\title{
AVALIAÇÃO EXPERIMENTAL DA RECUPERAÇÃO DO ÍLEO PARALÍTICO PÓS-OPERATÓRIO APÓS COLECTOMIA CONVENCIONAL E LAPAROSCÓPICA
}

\author{
EXPERIMENTAL EVALUATION OF THE POSTOPERATIVE ILEUS AFTER \\ CONVENTIONAL AND LAPAROSCOPIC COLECTOMY
}

\author{
Álvaro Antônio Bandeira Ferraz, TCBC-PE ${ }^{1}$ \\ Carlos Augusto Mathias ${ }^{1}$
}

\begin{abstract}
RESUMO: A cirurgia laparoscópica tem determinado reavaliações de muitos dogmas cirúrgicos. Dentre esses dogmas, está a aparente ausência de íleo paralítico na cirurgia laparoscópica. O objetivo do autor foi avaliar, de maneira experimental, em cães, as diferenças de recuperação da motilidade do cólon após uma colectomia tradicional e uma colectomia videolaparoscópica assistida, determinando ainda as características do íleo paralítico pós-operatório, em cães, após uma cirurgia laparoscópica. Foram utilizados dez cães mestiços, sadios, pesando de 20 a 30 kg, com idade entre 4 e 5 anos. Após uma noite de jejum, os eletrodos foram implantados, através de uma laparotomia, sob condições assépticas e anestesia geral. Cada animal teve implantado oito eletrodos bipolares. Quatro desses eletrodos foram implantados no intestino delgado, e os quatro eletrodos restantes, localizados no cólon, estando o mais distal a $20 \mathrm{~cm}$ da reflexão peritoneal do reto. Foi estabelecido um período de dez dias para a total recuperação do íleo paralítico pós-operatório. Após a aquisição de traçados controles, os animais foram randomizados para a realização de uma colectomia laparoscópica ou uma colectomia através de laparotomia. Após uma noite de jejum, a colectomia foi realizada sob condições assépticas e anestesia geral. Após a extubação iniciou-se a aquisição dos dados. A aquisição foi realizada até a completa recuperação do íleo paralítico, de maneira ininterrupta. Nenhum dos animais recebeu analgésicos no período pós-operatório. Não houve diferença entre o final da colectomia e o surgimento do primeiro complexo motor migrante (CMM), no intestino delgado. Com relação ao final das colectomias e o retorno à fase II, também não houve diferença estatisticamente significativa entre os dois grupos. Os intervalos de tempo entre as ressecções colônicas e o aparecimento da primeira contração colônica migrante (CCM) não foram estatisticamente diferentes, assim como não foram os intervalos entre o final da colectomia e o surgimento da primeira contração migratória gigante do cólon. Quando relacionamos o final da cirurgia e a primeira evacuação, não houve diferença entre os grupos. O autor concluiu que a colectomia laparoscópica cursa com íleo paralítico, e que não houve diferenças entre os grupos, com relação ao período de recuperação do íleo paralítico pós-operatório, nos cães estudados.
\end{abstract}

Unitermos: Íleo; Motilidade gastrointestinal; Complexo motor migrante; Motilidade colônica.

\section{INTRODUÇÃO}

A cirurgia videolaparoscópica tem apresentado uma evolução e uma expansão tão impressionante que qualquer matéria ou publicação sobre este assunto corre o risco de estar sendo obsoleto e ultrapassado.

A literatura tem descrito dois tipos de ressecção laparoscópica de segmentos de cólon. A primeira em que a anastomose do cólon é realizada fora da cavidade abdominal. É a chamada ressecção assistida. A outra maneira é aquela em que a anastomose colônica é realizada dentro da cavidade abdominal.
Nas colectomias assistidas, a laparoscopia é utilizada para realizar toda a liberação do órgão, ligando seus vasos mesentéricos. Após esta etapa, é realizada uma pequena incisão $(3-7 \mathrm{~cm})$, na qual o cólon é exposto, seccionado e realizada a anastomose, com ou sem grampeadores mecânicos. ${ }^{1}$

As ressecções colônicas, de maneira laparoscópica, implicam um período de internação menor, assim como menor dor referida no período pós-operatório., ${ }^{2,3} \mathrm{Na}$ casuística de Falk et al, ${ }^{2}$ os custos das ressecções colônicas através da laparoscopia foram mais elevados, quando comparados às colectomias abertas.

1. Professor Adjunto do Departamento de Cirurgia da Universidade Federal de Pernambuco.

Recebido em 26/4/99

Aceito para publicação em 4/10/99

Trabalho apresentado como requisito para a obtenção do Grau de Doutor em Medicina pela Universidade Federal de Pernambuco. 
O íleo paralítico pós-operatório é definido como sendo a paralização dos movimentos gastrointestinais, caracterizado por distensão abdominal e acúmulo de gás e fluidos na luz intestinal, após um procedimento cirúrgico. ${ }^{4} \mathrm{O}$ custo estimado do íleo paralítico é de cerca de U\$750.000.000,00, por ano, nos Estados Unidos. ${ }^{5}$ Sua etiologia continua indefinida. Sabese, porém, ser multifatorial, e que a hiperatividade simpática, a irritação do peritônio, os distúrbios metabólicos, comuns ao pós-operatório, efeitos de algumas drogas anestésicas e analgésicas contribuem de alguma forma para a presença e manutenção do íleo paralítico.

A administração de opiáceos, no alívio da dor, no pósoperatório imediato, prolonga o período de íleo paralítico. ${ }^{6,7,8}$ A administração de morfina resulta em contrações individuais não migratórias, no cólon direito e no cólon esquerdo, seguido de um efeito inibitório das contrações migratórias. ${ }^{6,8}$ Como a morfina e seus derivados são os principais analgésicos utilizados no pós-operatório, admite-se que o íleo paralítico venha sendo prolongado por conta dessa utilização.

A partir deste raciocínio, outras drogas têm sido estudadas com relação à analgesia pós-operatória e sua relação com a motilidade gastrointestinal. ${ }^{4}$ Os antiinflamatórios nãohormonais têm merecido uma atenção especial, principalmente porque, inibindo a ciclo-oxigenase, estariam inibindo a produção das prostaglandinas. ${ }^{9110,11}$ A inibição das prostaglandinas tem sido associada a um aumento da motilidade gastrointestinal. ${ }^{9,11}$ No entanto, a utilização de um antiinflamatório não-hormonal, o ketorolac tromethamine, não foi capaz de inibir a presença do íleo paralítico pós-operatório, mas, ao contrário da morfina, este não trouxe nenhum efeito deletério à recuperação dos movimentos colônicos após a cirurgia. ${ }^{4,9}$

O entendimento do íleo paralítico continua incompleto, mas, estudos clínicos e experimentais permitirão a compreensão de alguns pontos básicos: tório; 7,8

- os opiáceos prolongam o íleo paralítico pós-opera-

- o íleo paralítico não está relacionado à extensão e a duração da cirurgia; ${ }^{12}$

- o íleo paralítico parece ser fortemente influenciado por uma hiperatividade simpática; ${ }^{13,14,15}$

- o cólon esquerdo é o principal órgão alvo do íleo paralítico. ${ }^{16}$

A recente explosão da cirurgia laparoscópica tem resultado em reavaliações de, até então, antigos dogmas da cirurgia geral. Dentre esses dogmas cirúrgicos está a aparente ausência de íleo paralítico., ${ }^{2,3} \mathrm{O}$ objetivo deste trabalho foi avaliar, de maneira experimental, em cães, a recuperação da motilidade do cólon após uma colectomia videolaparoscópica assistida, comparando-a a uma colectomia tradicional.

\section{MATERIAL E MÉTODOS}

Na realização đeste estudo foram utilizados dez cães mestiços, pesando de 20 a $30 \mathrm{~kg}$, com idade entre 4 e 5 anos.
O protocolo de estudo foi analisado, julgado e aprovado pelo Animal Welfare Committee do Medical College of Wisconsin. Os animais foram mantidos no biotério do Medical College of Wisconsin, em jaulas individuais, e alimentados com ração própria para cães, uma vez ao dia, e água ad libitum.

Após uma noite de jejum, os eletrodos foram implantados, através de uma laparotomia, sob condições assépticas e anestesia geral. A anestesia geral foi realizada com fenobarbital $(25 \mathrm{mg} / \mathrm{kg})$ intravenoso, após uma pré-anestesia com ketamine $(10 \mathrm{mg} / \mathrm{kg})$, intramuscular. Foi utilizado antibiótico profilático, cefazolina, $100 \mathrm{mg} / \mathrm{kg}$, na indução anestésica. A incisão realizada foi uma paramediana esquerda, com cerca de $8 \mathrm{~cm}$ de comprimento. Cada animal teve implantado oito eletrodos, bipolares, de acordo com descrição anterior. ${ }^{17}$ Quatro destes eletrodos foram implantados no intestino delgado, sendo o primeiro distando $50 \mathrm{~cm}$ do ângulo de Treitz e os demais distalmente, em intervalos de $10 \mathrm{~cm}$. Os quatro eletrodos restantes, localizados no cólon, distavam $5 \mathrm{~cm}$ entre si, estando o mais distal a $20 \mathrm{~cm}$ da reflexão peritoneal do reto. A cavidade foi fechada com prolene 0 .

O eletrodo implantado fora também previamente descrito. ${ }^{17}$ Esses eletrodos foram suturados na serosa, borda contramesenterial, no intestino delgado e do cólon (tenia). Os eletrodos foram adaptados a uma cânula de metal, a qual era exteriorizada, através de uma incisão, no flanco esquerdo do animal. Após a implantação dos eletrodos, os animais foram recolocados nas suas respectivas jaulas.

Foi estabelecido um período de dez dias para a total recuperação do íleo paralítico pós-operatório.

Restabelecidos do íleo paralítico, os animais foram levados ao laboratório de análises para sessões de gravação da atividade elétrica, que serviram de controle. Foram realizadas três sessões, em três dias consecutivos, durando de seis a oito horas cada sessão. Neste primeiro momento era determinada a sensibilidade de cada eletrodo, em cada animal, injetando, por via subcutânea, $0,2 \mathrm{mg} / \mathrm{kg}$ de betanecol.

Os sinais elétricos foram gravados utilizando-se um polígrafo de oito canais. A resposta de freqüência do polígrafo foi de 0,02 a $15 \mathrm{~Hz}$. Os sinais foram simultaneamente registrados em um gravador de oito canais magnéticos, para posterior análise e para fins de arquivo.

Após a aquisição dos traçados controles, os animais foram randomizados para uma colectomia laparoscópica e uma colectomia por via aberta.

Após uma noite de jejum, a colectomia foi realizada sob condições assépticas e anestesia geral. Utilizou-se na anestesia geral fenobarbital $(35 \mathrm{mg} / \mathrm{kg})$ intravenoso, após uma préanestesia com ketamine $(10 \mathrm{mg} / \mathrm{kg})$, intramuscular. Foi utilizado antibiótico profilático, carbenicilina, $100 \mathrm{mg} / \mathrm{kg}$ e gentamicina, 1,5 mg/kg, na indução anestésica e oito horas após, de acordo com as recomendações do Animal Welfare Committee do Medical College of Wisconsin.

A colectomia aberta foi então procedida após uma incisão e, na anastomose, foram utilizados grampeadores mecâ- 
nicos lineares. A peça ressecada compreendia $20 \mathrm{~cm}$ de íleo terminal e dos $15 \mathrm{~cm}$ proximais do cólon. A cavidade foi novamente fechada com prolene 0 .

A colectomia videolaparoscópica foi realizada com a aposição de quatro trocartes de $10 \mathrm{~mm}$, após realização de pneumoperitônio com $\mathrm{CO}_{2}$ com agulha de Veress. A pressão intra-abdominal foi mantida abaixo de $14 \mathrm{mmHg}$. Na dissecção e delimitação do segmento a ser ressecado, foi utilizado bisturi elétrico bipolar, com posterior ligadura dos vasos sangüíneos com clips de titânio. Após a realização da dissecção e delimitação do segmento a ser ressecado, realizouse uma pequena incisão de cerca de $5 \mathrm{~cm}$ no flanco direito, em extensão do orifício do trocarte. O segmento a ser ressecado foi, então, exteriorizado, ressecado e anastomosado com grampeadores mecânicos lineares. Realizada a anastomose, a incisão foi fechada com prolene zero. A extensão do segmento ressecado foi similar ao grupo da colectomia aberta

Após a extubação, os animais foram transferidos ao laboratório de análises e conectados ao polígrafo, para início da aquisição dos dados, que se deu até a completa recuperação do íleo paralítico, de maneira ininterrupta. Os cães tinham acesso a água ad libitum e só reiniciavam dieta normal após a recuperação do íleo paralítico.

Nenhum animal recebeu analgésico no período pósoperatório.

Uma contração era considerada positiva quando apresentava uma deflexão acima de $5 \mathrm{~mm}$ e com duração superior a 3 segundos. Com isto, eliminamos artefatos próprios da respiração e dos movimentos voluntários e involuntários dos animais.

Um ciclo do complexo motor migratório é dividido em quatro fases, ${ }^{18}$ a saber:

Fase I - Sem contrações musculares. As ondas lentas persistem;

Fase II - Aparecem contrações aglomeradas e irregularmente espaçadas. Aumento na freqüência, em direção da fase III;

Fase III - Freqüência máxima das contrações, cada onda lenta apresenta uma contração;

Fase IV- Redução na freqüência das contrações na direção da fase I.

A contração colônica migrante foi definida como sendo uma contração colônica, em, no mínimo, três sítios de gravação, migrando em velocidade constante. Já a contração migratória gigante foi definida como sendo um conjunto de contrações (mais de três) em, no mínimo, três sítios de gravação, migrando sobre uma velocidade constante.

O período controle e o período pós-colectomia foi analisado para:

- duração e velocidade das CMM (Complexo Motor Migrante )

- número de contrações colônicas

- intervalo de tempo entre as colectomias e:

- o primeiro CMM
- o retorno à fase II

- primeira CCM (Contração Colônica Migrante)

- primeira CMG (Contração Migratória Gigante)

- primeira evacuação

Após completados os estudos, os animais foram mortos com uma solução de fenobarbital e fenitoína, injetados intravenosamente.

Os dados foram analisados de maneira estatística. Nos casos em que os dados numéricos dos grupos indicassem um afastamento da curva de normalidade realizamos um teste não-paramétrico (Teste de Friedman). Nos casos em que a distribuição dos dados garantiu uma linearidade com a curva normal realizou-se o teste de " $t$ " de Student, bicaudal, assumindo variâncias diferentes.

Um valor de $\mathrm{p}<0,05$ foi considerado indicativo de diferença estatisticamente significativa.

\section{RESULTADOS}

Todos os cães toleraram bem as laparotomias, tanto para implante dos eletrodos quanto para a realização das colectomias. Não houve complicações relacionadas aos procedimentos cirúrgicos.

Não houve diferença com relação entre o final da colectomia e o surgimento do primeiro CMM. A média nos cães submetidos à colectomia aberta foi de 388,8 minutos, enquanto nos animais submetidos a colectomia laparoscópica assistida este intervalo de tempo foi de 453,6 minutos. Esta diferença não foi estatisticamente significativa (Tabela 1).

Com relação entre o final das colectomias e o retorno à fase II também não houve diferença estatisticamente significativa entre os dois grupos. Nos cães submetidos à colectomia aberta, este retorno se deu, em média, 2.544 minutos após o término da cirurgia, comparados aos 1.958,4 minutos do grupo das colectomias laparoscópicas (Tabela 2).

O intervalo de tempo entre as ressecções colônicas e o aparecimento da primeira contração colônica migrante (CCM) não foi estatisticamente diferente (Tabela 3). Na tabela 4 estão

Tabela 1

Intervalo de tempo entre o final da colectomia e o primeiro Complexo Motor Migrante (CMM)

\begin{tabular}{l|c|c}
\hline Cão & $\begin{array}{c}\text { Colectomia aberta } \\
\text { (minutos) }\end{array}$ & $\begin{array}{c}\text { Colectomia laparoscópica } \\
\text { (minutos) }\end{array}$ \\
\hline 1 & 793 & 291 \\
2 & 328 & 450 \\
3 & 260 & 540 \\
4 & 377 & 325 \\
5 & 186 & 662 \\
Média & 388,80 & 453,60 \\
Desvio padrão & 237,13 & 153,20 \\
Erro padrão & 106,05 & 68,51 \\
\hline
\end{tabular}

Análise estatística:

Friedman's Chi-square $=0,20$

$$
p>0,05
$$


Tabela 2

Intervalo de tempo entre o final da colectomia e o retorno à fase II

\begin{tabular}{l|c|c}
\hline Cão & $\begin{array}{c}\text { Colectomia aberta } \\
\text { (minutos) }\end{array}$ & $\begin{array}{c}\text { Colectomia laparoscópica } \\
\text { (minutos) }\end{array}$ \\
\hline 1 & 3384 & 2616 \\
2 & 2376 & 1296 \\
3 & 1572 & 1728 \\
4 & 2676 & 2736 \\
5 & 2712 & 1416 \\
Média & 2544,00 & 1958,40 \\
Desvio padrão & 656,55 & 675,12 \\
Erro padrão & 293,62 & 301,92 \\
\hline
\end{tabular}

Análise estatística: $t=1,98$

$$
p>0,05
$$

Tabela 3

Intervalo de tempo entre o final da colectomia e a primeira Contração Colônica Migratória (CCM)

\begin{tabular}{l|c|c}
\hline Cão & $\begin{array}{c}\text { Colectomia aberta } \\
\text { (minutos) }\end{array}$ & $\begin{array}{c}\text { Colectomia laparoscópica } \\
\text { (minutos) }\end{array}$ \\
\hline 1 & 126 & 140 \\
2 & 338 & 532 \\
3 & 131 & 199 \\
4 & 302 & 325 \\
5 & 947 & 591 \\
Média & 368,80 & 357,40 \\
Desvio padrão & 337,40 & 199,00 \\
Erro padrão & 150,90 & 89,00 \\
\hline
\end{tabular}

Análise estatística: Friedman's Chi-square $=1,80$

$$
p>0,05
$$

expostos os intervalos entre o final da colectomia e o surgimento da primeira contração migratória gigante do cólon. Não houve diferença estatística entre os grupos.

Quando se relacionou o final da cirurgia com a primeira evacuação, não houve diferenças entre os grupos (Tabela 5). Os cães submetidos à colectomia aberta defecaram, em média, após 2.715,8 minutos do término da cirurgia. Já no grupo de cães submetidos a colectomia laparoscópica, a defecação se deu, em média, com 2.215,2 minutos após o término da cirurgia.

$\mathrm{Na}$ análise da duração do complexo motor migrante (CMM) não houve diferença estatística entre os grupos.

O grupo de cães submetidos à colectomia laparoscópica apresentou um CMM estatisticamente mais lento quando comparado ao grupo de cães submetidos à colectomia aberta, e quando comparado ao seu mesmo grupo, no período controle.

A velocidade média ( $\mathrm{cm} / \mathrm{min}$ ) dos CMM, no período controle e no estado pós-colectomia foi semelhante, sem diferenças estatísticas, quando comparados os grupos de animais submetidos à colectomia aberta e os submetidos à colectomia laparoscópica .

O número de contrações colônicas no período controle foi semelhante entre os dois grupos. Porém, após a realização da colectomia, o grupo de cães submetidos ao procedimento laparoscópico apresentou uma diminuição no número destas contrações estatisticamente significativa. Este número tam-
Tabela 4

Intervalo de tempo entre o final da colectomia e a primeira Contração Migratória Gigante do Cólon (CMG)

\begin{tabular}{l|c|c}
\hline Cão & $\begin{array}{c}\text { Colectomia aberta } \\
\text { (minutos) }\end{array}$ & $\begin{array}{c}\text { Colectomia laparoscópica } \\
\text { (minutos) }\end{array}$ \\
\hline 1 & 3412 & 2715 \\
2 & 2700 & 1513 \\
3 & 1602 & 1790 \\
4 & 2150 & 3000 \\
5 & 2989 & 1516 \\
Média & 2570,60 & 2106,80 \\
Desvio padrão & 709,70 & 701,70 \\
Erro padrão & 317,40 & 313,80 \\
\hline
\end{tabular}

Análise estatística: $t=1,03$

$$
p>0,05
$$

Tabela 5

Intervalo de tempo entre o final da colectomia e a primeira evacuação

\begin{tabular}{l|c|c}
\hline Cão & $\begin{array}{c}\text { Colectomia aberta } \\
\text { (minutos) }\end{array}$ & $\begin{array}{c}\text { Colectomia laparoscópica } \\
\text { (minutos) }\end{array}$ \\
\hline 1 & 3536 & 2816 \\
2 & 2717 & 1540 \\
3 & 1615 & 1796 \\
4 & 2699 & 3009 \\
5 & 3012 & 1915 \\
Média & 2715,80 & 2215,20 \\
Desvio padrão & 702,20 & 654,40 \\
Erro padrão & 314,00 & 292,60 \\
\hline
\end{tabular}

Análise estatística: $t=1,16$

$$
p>0,05
$$

bém teve representatividade estatística, quando comparamos os período controle e o pós-colectomia do grupo de laparoscopia. O número de contrações no período controle foi de 24,85 /hora, comparados com os 18,8 /hora do período póscolectomia.

\section{DISCUSSÃo}

No cólon são identificados, através da eletromiografia, quatro tipos de atividade elétrica: ${ }^{19,20}$

- atividade elétrica de controle;

- atividade elétrica de resposta de curta duração;

- atividade elétrica de resposta de longa duração; e

- complexo elétrico contráctil.

Por questões de terminologia, e de acordo com os nossos objetivos, avaliamos, no cólon, apenas a atividade elétrica de resposta (contração colônica) e o complexo elétrico contráctil, durante uma migração (contração migratória gigante).

É importante salientar, no entanto, que a tradução mecânica de uma atividade elétrica de resposta é uma contração. ${ }^{19,20}$ Esta relação é de $1: 1 \cdot{ }^{19,20}$

A determinação do número de animais a serem utilizados foi cuidadosamente analisada. Arbitrariamente, assumimos um $\alpha=0,05$ e um $\beta=0,2$, e determinamos, ainda, que a 
diferença numérica entre os grupos se tornaria significativa, se excedesse 12 horas. Sendo assim, usando fórmulas estatísticas para cálculo de $\mathrm{n}$, determinamos o tamanho da amostra. O número de cinco animais, em cada grupo, mostrou ser suficiente para os objetivos determinados pelo autor.

$\mathrm{O}$ crescente interesse pela cirurgia laparoscópica foi inspirado, principalmente, por um número de vantagens desse tipo de procedimento em relação à cirurgia convencional: os pacientes apresentam menos dor pós-operatória; as cicatrizes, além de mínimas, são cosmeticamente aceitáveis; o período de internação hospitalar é menor e os pacientes retornam às suas atividades normais mais precocemente. ${ }^{1,2,21}$ No entanto, algumas conclusões com respeito à cirurgia laparoscópica, incluindo a ausência de íleo paralítico, são baseadas em observações clínicas sem respaldo científico.

O íleo paralítico tem uma correlação mínima com o estômago e o intestino delgado. ${ }^{16} \mathrm{O}$ íleo paralítico é decorrente de alterações no cólon esquerdo. ${ }^{16}$ Assim sendo, não se deve estudar íleo paralítico pós-operatório em lugares que não sejam o cólon esquerdo.

No presente estudo, o CMM no intestino delgado voltou aos padrões normais em intervalos de tempo semelhantes, quando comparamos os dois grupos (Tabela 1). Antes de se comentarem os resultados da tabela 2 , é necessário enfatizar alguns estudos sobre a fase II e a motilidade intestinal.

Lang et al, ${ }^{22}$ estudando a geração do CMM de cães, concluíram que muito mais do que um simples componente das fases do CMM, a fase II constituiria a atividade espontânea do intestino delgado. Durcef et $\mathrm{al}^{23}$ postularam ainda que o reaparecimento da fase II era seguida de uma progressiva coordenação e reorganização dos movimentos do tracto gastrointestinal.

A ausência da fase II pode estar relacionada com o íleo paralítico.

Nas peritonites, apesar do intestino delgado não estar paralisado, a fase III está presente, a fase II desaparece, acarretando uma desorganização das ondas propulsivas do intestino delgado, alterando a propulsão mecânica do tracto gastrointestinal. ${ }^{24}$

Em nosso trabalho, notamos uma ausência da fase II no primeiro dia de pós-operatório (Tabela 2) e um íntimo relacionamento com a primeira CMG e a primeira evacuação (Tabelas 4 e 5). Ou seja, a restauração da fase II coincidiu com a primeira evacuação após a cirurgia. Isto não significa, portanto, que a fase II seja o parâmetro de restauração do íleo paralítico. Íleo paralítico pós-operatório é definido como sendo uma paralisação dos movimentos gastrointestinais, caracterizados pelo acúmulo de gás e fluidos na luz intestinal, impedindo a ingesta oral de alimentos, após um ato cirúrgico. ${ }^{4} \mathrm{~A}$ sua recuperação está relacionada ao aparecimento de contrações migratórias no cólon esquerdo. ${ }^{6} \mathrm{O}$ fato do paciente não defecar não significa que ele permaneça em íleo.

Em ambos os grupos, o aparecimento da primeira contração colônica migratória $(\mathrm{CCM})$ se deu em torno da sexta hora de pós-operatório (Tabela 3), não havendo, portanto, diferenças entre os grupos.

O aparecimento da contração migratória gigante (CMG), melhor expressão da resolução do íleo paralítico, se deu de maneira semelhante nos dois grupos durante o segundo dia de pós-operatório (Tabela 4).

A contração migratória gigante (CMG), ou contração propulsiva, são contrações de alta amplitude e longa duração, que fazem migrar o bolo fecal. Estas contrações resultam em movimentos de massa, flatulência e defecação. ${ }^{19,20}$

A CMG ocorre de maneira involuntária, e o estímulo para o seu início ainda não foi determinado.

A observação de que pacientes submetidos a procedimentos laparoscópicos sejam capazes de se alimentar mais precocemente pode estar associada à possibilidade desses pacientes apresentarem menos dor pós-operatória e, conseqüentemente, terem menos necessidade de fazerem uso de opiáceos, ou qualquer outro tipo de analgésico.

Trabalhos realizados demonstraram, em macacos e em humanos, ${ }^{7,8}$ que a utilização de narcóticos interrompem a recuperação normal do íleo paralítico. Morfina administrada de maneira parenteral induz contrações fásicas, não-migratórias, em todo o cólon, principalmente no cólon esquerdo. ${ }^{7,8}$ Em nosso estudo, no período pós-operatório não foram utilizados analgésicos, ou qualquer outra droga que pudesse interferir com a recuperação do íleo. A utilização de antiinflamatórios não-hormonais apresenta uma ação, ainda duvidosa, na motilidade do cólon, principalmente aqueles que inibem a síntese das prostaglandinas. ${ }^{4,9,11,25,26}$

Inúmeras são as publicações documentando que os pacientes submetidos a procedimentos laparoscópicos utilizam uma quantidade de analgésicos menor do que aqueles submetidos a procedimentos abertos. ${ }^{2,21,27}$

Soper et al $^{28}$ não utilizaram narcóticos no pós-operatório imediato de pacientes submetidos a colecistectomia laparoscópica. Em contrapartida, nos pacientes submetidos a colecistectomia aberta, utilizaram em média $35 \mathrm{mg}$ de morfina no primeiro dia de pós-operatório, para alívio da dor. Na série publicada por Schirmer et al, ${ }^{29} 36 \%$ dos pacientes submetidos a colecistectomia laparoscópica não utilizaram analgésicos no pós-operatório, $25 \%$ utilizaram apenas analgésicos orais e $39 \%$ necessitaram de morfina intravenosa para alívio da dor. Dos 13 pacientes submetidos a colecistectomia aberta, 11 fizeram uso de bomba de infusão de morfina durante os dois primeiros dias após a cirurgia.

Vạllina et $\mathrm{al}^{30}$ comparando apendicectomias laparoscópicas com apendicectomias convencionais, também notaram que $31 \%$ dos pacientes submetidos ao procedimento laparoscópico não usaram analgésicos e $25 \%$ dos pacientes só utilizaram analgésicos orais. Glaser et $\mathrm{al}^{27}$ também identificaram, em trabalho publicado em 1995, que, em média, os pacientes submetidos a colecistectomias convencionais utilizaram 15,3 mg de morfina, comparando aos 4,1 mg dos pacientes submetidos a colecistectomia laparoscópica $(\mathrm{p}<0,001)$. 
Pacientes que apresentam menos dor pós-operatória, conseqüentemente apresentam menos stress.

Existem fortes evidências de que a hiperatividade simpática exerce função primordial na fisiopatologia do íleo paralítico. Alguns autores chegaram a publicar que o bloqueio do sistema adrenérgico resultaria na supressão do íleo paralítico..$^{13,14,15}$ A inervação simpática do intestino delgado e do cólon consiste em neurônios colinérgicos pré-ganglionares e em neurônios adrenérgicos pós-ganglionares. ${ }^{3}$ A maioria dos neurônios pós-ganglionares se origina nos gânglios prévertebrais. ${ }^{14,19-32}$ As fibras nervosas dos neurônios adrenérgicos pós-ganglionares inervam os plexos mioentérico e submucoso, além dos vasos sangüíneos do intestino delgado e do cólon. ${ }^{31,32}$ Estes nervos atuam, primariamente, modulando os neurônios colinérgicos excitatórios, inibindo a liberação de acetilcolina. ${ }^{32}$ Esta ação da norepinefrina nos neurônios colinérgicos é, supostamente, mediada por receptores alfa 1 e alfa 2 adrenérgicos. ${ }^{13,14,15,32}$

Em trabalhos realizados no laboratório do Departamento de Cirurgia da Universidade de Wisconsin foi sugerido que o sistema nervoso simpático adrenérgico era o principal responsável pela inibição da atividade contráctil do cólon esquerdo no período de pós-operatório. ${ }^{14,15}$ Nestes experimentos, a infusão intravenosa de fentolamina (bloqueador alfa adrenérgico), após quatro horas do término da cirurgia, restaurou, no cólon esquerdo, a atividade contráctil para níveis dentro da normalidade. Este achado não se repetiu no cólon direito. ${ }^{15}$
O cólon direito respondeu com um aumento da atividade contráctil, mas ainda significativamente abaixo do normal. Esses dados sugerem que o cólon esquerdo é mais sensível à ação adrenérgica do que o cólon direito.

Devido ao fato do sistema nervoso adrenérgico receber fibras oriundas de gânglios centrais e do sistema aferente da parede do intestino, não se sabe, ao certo, se a inibição das contrações no período pós-operatório é mediada centralmente, na periferia ou se por ambos. ${ }^{20}$

Os níveis de epinefrina e norepinefrina estão elevados durante o primeiro dia de pós-operatório (DPO) e vão progressivamente retornando aos níveis normais, alcançando a normalidade do terceiro ao quinto DPO. Este retorno aos níveis normais coincide com a restauração da atividade motora do cólon. ${ }^{33}$ Catecolaminas séricas elevadas, por si só, não causam íleo paralítico. ${ }^{32}$ Níveis séricos elevados são evidenciados em pacientes com feocromocitoma e em grandes queimados, e o íleo paralítico não se relaciona com seus níveis séricos. ${ }^{14.15}$

O trabalho publicado por Glaser et $\mathrm{al}^{27}$ evidenciou níveis de epinefrina e norepinefrina nos pacientes submetidos a colecistectomia laparoscópica, estatisticamente mais elevados dos que aqueles submetidos a colecistectomia laparoscópica. Os níveis plasmáticos de ACTH, hormônio adrenocorticotrófico, e cortisol não foram estatisticamente diferentes.

O problema do íleo paralítico é multifatorial e, a despeito dos recentes avanços, ainda não foi desvendada a maneira de se eliminar o íleo paralítico pós-operatório.

\begin{abstract}
Laparoscopic surgery has gained a wide acceptance, determining a review in many surgical dogmas. One of those dogmas is the apparent lack of postoperative ileus. The patterns and the difference between laparoscopic assisted colectomy and the traditional open colectomy, of colonic motility were determined experimentally. Ten, healthy, dogs, weighting 20 to $30 \mathrm{~kg}$, were instrumented with bipolar electrodes. Each animal was instrumented with 8 bipolar recording electrodes, 4 on the small bowel and 4 on the colon. Dogs were allowed to recover for ten days, when baseline myoelectric recordings were obtained. Then, the dogs were randomized to open or laparocopic colectomy. Operations were carried out after an overnight fast, under sterile conditions and general anesthesia. Myoelectric recording resumed immediately following operations and was continued until the complete resolution of the postoperative ileus. None of the dogs received analgesics. There were no significant differences between the groups regarding the end of the colectomy and the first Migrating Myoelectric Complex (MMC), the end of the colectomies and the return of the phase II, the time division between the end of the colectomies and the first Migrating Colonic Contraction, the end of the colectomies and the first Giant Migrating Colonic Contraction, and the relation between the end of the colectomy and the first evacuation. The authors concluded that laparoscopic assisted colectomy produces postoperative ileus and there was no difference between the groups, relating the period of time of recovery from postoperative paralytic ileus.
\end{abstract}

Key Words: Ileus; Gastrointestinal motility; Migrating myoelectric complex; Colonic motility.

\title{
REFERÊNCIAS
}

1. Hoffman GC, Baker JW, Fitchett CW, et al - Laparoscopic-Assisted colectomy: Initial experience. Ann Surg 1994;219:732-743.
2. Falk PM, Beart RW, Wexner SD, et al - Laparoscopic colectomy: a critical appraisal. Dis Colon Rectum 1993;1:28-34. 
3. Soper NJ, Brunt LM, Kerbl K - Laparoscopic general surgery. N Eng J Med 1994;330:409-419.

4. Ferraz $\mathrm{AAB}$ - Atividade mioelétrica do cólon: avaliação experimental e clínica do ketorolac tromethamine, durante e após a recuperação do íleo paralítico pós-operatório. Tese para obtenção do grau de Mestre em Cirurgia do CCS da UFPE. 1994

5. Moss G, Regal ME \& Lichtifg LK - Reducing postoperative pain, narcotics and lenght of hospitalization. Surgery 1986; 90:206-210.

6. Condon RE, Frantzides CT, Cowles V, et al - Resolution of postoperative ileus in humans. Ann Surg 1986;203:574-581.

7. Frantzides CT, Condon RE, Schulte WJ, et al - Effects of morphine on colonic myoeletric and motor activity in subhuman primates. Am J Physiol 1990;258(21):G247-252

8. Frantzides CT, Cowles V, Salaymeh B, et al - Morphine effects on human colonic myoeletric activity in the postoperative period. Am J Surg 1992; 163:144-149.

9. Ferraz AAB - Atividade mioelétrica do cólon: avaliação experimental e clínica do ketorolac tromethamine, durante e após a recuperação do íleo paralítico pós-operatório. An Fac Med Univ Fed Pernamb 1994; 39:47-56.

10. Fragen RJ - Ketorolac: non-opioid analgesic. Comments. Curr Surg 1993; 50(2):130-131.

11. Sanders KM - Endougenous prostaglandin E and contractile activity of isolated ileal smooth muscle. Am J Physiol 1978; 234:E209-E212.

12. Graber JN, Schulte WJ, Condon RE, et al - Relationship of duration of postoperative ileus to extent and site of operative dissection. Surgery 1982; 92:87-92.

13. Catchpole BN - Ileus: use of sympathetic blocking agents in its treatment Surgery 1969;66:811-820.

14. Cowles VE, Zhu YR, Herranz E, et al - Adrenergic antagonists and postoperative ileus. Gastroenterology 1990; 99:1210.

15. Esser MJ, Mahoney JL, Robinson JC, et al - Effect of adrenergic agents on colonic motility. Surgery 1987; 102:416-423.

16. Woods JH, Erickson LW, Condon RE, et al - Postoperative ileus: a colonic problem ? Surgery 1978; 84:527-533.

17. Frantzides CT, Matsumoto T, Lang I, et al - An intrinsic neural pathway for long intestino-intestinal inhibitory reflexes. Gastroenterology 1987; 92:594-603.

18. Zenilman ME - Origem e controle da motilidade gastrointestinal. Clin Cir Am Norte 1993; 6:1115:1135.

19. SarnaSK - Physiology and pathophysiology of colonic motor activity. Part one of two. Dig Dis Sci 1991; 36:827-862

20. Sarna SK -Physiology and pathophysiology of colonic motor activity. Part two of two. Dig Dis Sci 1991; 36:998-1018.

21. Soper NJ - Laparoscopic general surgery - past, present and future Surgery 1993;113:1-3.
22. Lang IM, Sarna SK \& Condon RE - Generation of phase I and II of migrating myoelectric complex in the dog. Am JPhysiol 1986; 251: G201-G207.

23. Ducerf C, Duchamp C \& Pouyet M - Postoperative electromyographic profile in human jejunum. Ann Surg 1992; 215:237-243.

24. Mathias CAM-Atividade mioelétrica do intestino delgado na infecção peritoneal experimental. 1993. Tese (Mestrado), Centro de Ciências da Saúde - UFPE. Recife.

25. Coelho JCU, Gouma DJ, Li YF, et al - Effect of 16,16-Dimethyl Prostaglandin $\mathrm{E}_{2}$ on the myoelectric activity of the gastrointestinal tract of the opossum. J Physiol 1986; 377:421-427.

26. Thor P, Konturek JW, Konturek SJ, et al - Role of prostaglandins in control of intestinal motility. Am J Physiol 1985; 248:G353-G359.

27. Glaser F, Sannwald GA, Buhr HJ, et al - General stress response to conventional and laparoscopic cholecistectomy. Ann Surg 1995; 221:372-380.

28. Soper NJ, Barteau JA, Clayman RV, et al - Comparasion of early postoperative results for laparoscopic versus standard open cholecystectomy. Surg Gynec Obstet 1992;174:114-118.

29. Schirmer BD, Edge SB, Dix J, et al - Laparoscopic cholecystectomy: treatment of choice foi symptomatic cholelithiasis. Ann Surg 1991; 213:665-676.

30. Vallina VL, Velasco JM \& McCulloch CS - Laparoscopic versus conventional appendectomy. Ann Surg 1993; 218:685-692.

31. Bennett MR \& Rogers DC - A study of the innervation of the taenia coli. J Cell Biol 1967; 33:573-596.

32. Sino N, NakazatoY \& Ohga A - Presynaptic inhibiting effects of catecholamines on cholinergic transmission in the smooth muscle of the chick stomach. Eur J Pharmacol 1978;51:229-237.

33. Tarnoky K, Szenohradszky J \& Petri G - Plasma cathecholamine levels in the postoperative period in complication-free and "paralitic" ileus patients. Acta Chir Hung 1987;28:287-289.

\section{ENDEREÇO PARA CORRESPONDÊNCIA}

Dr. Álvaro Antônio Bandeira Ferraz

Av. Rosa e Silva, 2063

50050-020 - Recife - PE 